\title{
SECAGEM DE CORNICHÃO (Lotus corniculatus L.) EM LEITO FIXO COM ESCOAMENTO DE AR PARALELO
}

\author{
A. F. KANAAN ${ }^{2}$, C. G. FLORES ${ }^{1}$, J.C.P. OLIVEIRA ${ }^{3}$ e A.R.F. ALMEIDA Al, $^{1, *}$ \\ ${ }^{1}$ Universidade Federal do Pampa, Curso de Engenharia Química, Campus Bagé \\ ${ }^{2}$ Universidade Federal do Pampa, Programa de Pós-Graduação em Engenharia, Campus \\ Alegrete e Bagé \\ ${ }^{3}$ Empresa Brasileira de Pesquisa Agropecuária, Embrapa Pecuária Sul \\ *e-mail para contato: andre.almeida@unipampa.edu.br
}

\begin{abstract}
RESUMO - Este trabalho teve como objetivo realizar um estudo sobre o processo de secagem de sementes de cornichão (Lotus corniculatus L.) em um secador de leito fixo com fluxo de ar paralelo. Para alcançar este objetivo, o trabalho foi dividido nas seguintes etapas: caracterização física e fisiológica das sementes, análise do processo de reumidificação das sementes, estudo das isotermas de dessorção das sementes reumidificadas e avaliação dos efeitos da variação da temperatura, da velocidade do ar e da altura da bandeja sobre a cinética de secagem e qualidade fisiológica do produto final. Os dados experimentais obtidos mostraram que as condições de secagem podem influenciar significativamente a umidade de armazenamento e a qualidade fisiológica do produto final, refletida nos valores de germinação total.
\end{abstract}

\section{INTRODUÇÃO}

No Rio Grande do Sul, aproximadamente $61 \%$ da cobertura vegetal é composta por pastagens nativas as quais apresentam um crescimento vegetativo em condições de clima quente. Porém, as estações de clima frio são as mais predominantes no estado e isto faz com que ocorra uma diminuição na produção de pastagens (Mota et al., 1981). Para contornar este quadro, torna-se necessária à implementação de forrageiras adaptadas ao clima frio. Dentre as forrageiras mais adaptáveis aos períodos de clima frio tem-se como destaque o cornichão (Lotus corniculatus L.), que é leguminosa forrageira hibernal utilizada em ampla escala no Rio Grande do Sul a qual se destaca pela sua boa capacidade colonizadora, sua permissão para ser empregada em consórcio com demais leguminosas e pela sua tolerância à acidez e à baixa fertilidade do solo. (Melo e Barros, 2003).

Curtis e McKersie (1984) informa que, apesar de suas qualidades, há alguns fatores negativos que influenciam o estabelecimento e persistência no cultivo do cornichão. Tais fatores podem ser atribuídos a sua suscetibilidade a agentes patogênicos causadores de doenças nas raízes e coroas (Viands et al., 1994), ao seu lento crescimento inicial e à baixa qualidade da semente (Ettlin e Laverack, 1996). No que se refere à qualidade das sementes, o fator limitante se deve a baixa germinação. De modo geral os valores encontrados para o cornichão (Lotus corniculatus L.) são de aproximadamente $60 \%$, valor considerado baixo e que está vinculado ao processo de beneficiamento que tem como etapa limitante a secagem. 
O termo secagem pode ser definido com a remoção de líquido de um sólido por evaporação. Sendo assim, o material úmido que entra em contato com o ar insaturado sofre uma transferência simultânea de calor e de massa promovendo uma diminuição do conteúdo de umidade do material e a umidificação do ar. Dois estágios definem o processo total de secagem, o aquecimento do material e a evaporação de umidade até alcançar a umidade de equilíbrio (Strumillo e Kudra, 1986). Segundo Ferrari Filho (2011) as principais vantagens da secagem são a possibilidade de antecipação da colheita e redução da perda no campo ocasionada por condições e alterações climáticas e/ou pela ação de pragas e insetos. Ainda segundo este autor, a colheita antecipada também possibilita aos produtores obterem melhores preços pelos produtos quando a comercialização ocorrer em épocas de entressafra.

Goneli (2008) cita que a vida útil de produtos da agroindústria está diretamente relacionada com os processos de secagem e de armazenamento. Isso torna de suma importância o conhecimento da relação entre a umidade do material e a umidade relativa do ar. Neste contexto, a literatura (Samaniego-Esguerra et al., 1991; Morey et al., 1995; Chen e Jayas, 1998) afirma que os valores de umidade de equilíbrio dos produtos biológicos dependem da espécie, da variedade, do teor de umidade inicial e principalmente da temperatura e umidade relativa do ambiente em que se encontram. Para Figueirêdo e Queiroz (2007) o estudo da atividade de água de materiais pode ser feito mediante a avaliação de isotermas de sorção. Segundo Karathanos et al., (1996) as isotermas de sorção correspondem a uma relação física entre a umidade do material e a umidade realtiva correspondente a uma dada temperatura.

Apesar de o cornichão (Lotus corniculatus L.) ser uma forrageira bem difundida no mercado, a literatura ainda não fornece informações técnicas sobre suas propriedades físicas e fisiológicas, isotermas de dessorção e cinética de secagem. Estudos sobre esses parâmetros são necessários para otimizar o processo de beneficiamento deste material com o intuito de permitir a obtenção de um produto com melhor qualidade, menor custo e que satisfaça as exigências dos consumidores. Devido a isto, este trabalho teve como objetivo principal caracterizar física e fisiologicamente e estudar as isotermas de dessorção e a secagem de sementes de cornichão (Lotus corniculatus L.) em secador de leito fixo com escoamento de ar paralelo.

\section{MATERIAIS E MÉTODOS}

As sementes de cornichão (Lotus corniculatus L.) utilizadas na realização deste trabalho foram fornecidas pela Empresa Brasileira de Pesquisa Agropecuária - Embrapa Pecuária Sul da cidade de Bagé/RS. Todos os experimentos foram realizados a partir de um mesmo lote de sementes com o intuito de garantir um material com mesmas características físicas e fisiológicas iniciais. A caracterização física das sementes foi feita através das técnicas de picnometria gasosa para determinar a massa específica real $\left(\rho_{r}\right)$, ensaio de proveta para determinar a massa específica bulk $\left(\rho_{b}\right)$, ensaio de funil fixo para determinar o ângulo de repouso $(\theta)$, peneiramento para determinar o diâmetro médio de $\operatorname{Sauter}\left(d_{s}\right)$ e paquimetria para determinar o fator de forma esfericidade $(\phi)$. A porosidade do leito de sólidos $(\varepsilon)$ foi determinada pela relação entre a massa específica real e a massa específica bulk. Para a caracterização fisiológica foram efetuados testes de germinação seguindo a metodologia recomendada nas Regras para Análise de Sementes (Brasil., 1992). Esta análise constituiu na utilização de caixas gerbox juntamente com papel filtro, onde foram colocadas 100 sementes. 
As amostras foram submetidas a um confinamento controlado em uma estuda germinadora, operando na temperatura de $20{ }^{\circ} \mathrm{C}$ na influência de luz solar natural. As contagens da germinação $(G)$ foram procedidas no quarto e décimo segundo dia após o início do teste.

As sementes de cornichão, fornecidas pela Embrapa - Pecuária Sul, já haviam sido beneficiadas e tratadas para as condições ideais de mercado. Sendo assim, para a realização deste trabalho, foi necessário submetê-las a um processo de reumidificação. O processo de reumidificação utilizado neste trabalho foi embasado na metodologia de saturação proposta por Arnost Jr. (1997), a qual consistiu em expor as sementes em um ambiente fechado e saturado na temperatura de $40{ }^{\circ} \mathrm{C}$ por um intervalo de tempo de $24 \mathrm{~h}$. Terminado o processo de reumidificação, foi determinado o teor de umidade das sementes $\left(U_{B U}\right)$ através do método gravimétrico recomendado pela Association of Oficial Analytical Chemists - AOAC (1997).

As isotermas de dessorção foram determinadas pelo método gravimétrico estático, utilizando-se soluções de ácido sulfúrico em onze diferentes concentrações que garantiram a variação da umidade relativa do ar de 5 a $89 \%$. Os experimentos foram conduzidos nas temperaturas $(T)$ de 40,50 e $60{ }^{\circ} \mathrm{C}$. Cada análise foi realizada em triplicata, utilizando-se recipientes de vidros hermeticamente fechados, suporte e cadinho de plástico. No interior de cada recipiente foram inseridos $30 \mathrm{~mL}$ das soluções de ácido sulfúrico. Também foi inserido em cada cadinho de plástico aproximadamente $2,5 \mathrm{~g}$ de sementes de cornichão reumidificado. Os recipientes de vidros hermeticamente fechados foram colocados em estufa de circulação forçada nas temperaturas de 40,50 e $60{ }^{\circ} \mathrm{C}$, sendo realizadas pesagens do cadinho plástico no primeiro, sétimo, décimo e décimo quarto dia de experimento. Essas pesagens foram feitas com o objetivo de verificar o equilíbrio mássico para cada atmosfera estudada. Com o equilíbrio mássico alcançado no décimo quarto dia, determinou-se a $U_{B U}$ das sementes através do método gravimétrico recomendado pela AOAC (1997). Os resultados obtidos nas isotermas foram analisados através do ajuste dos dados experimentais aos modelos de BET, GAB, Oswin, Peleg e Hederson.

Para a etapa de secagem, foi utilizado o secador de leito fixo com escoamento de ar paralelo ilustrado na fotografia da Figura 1.

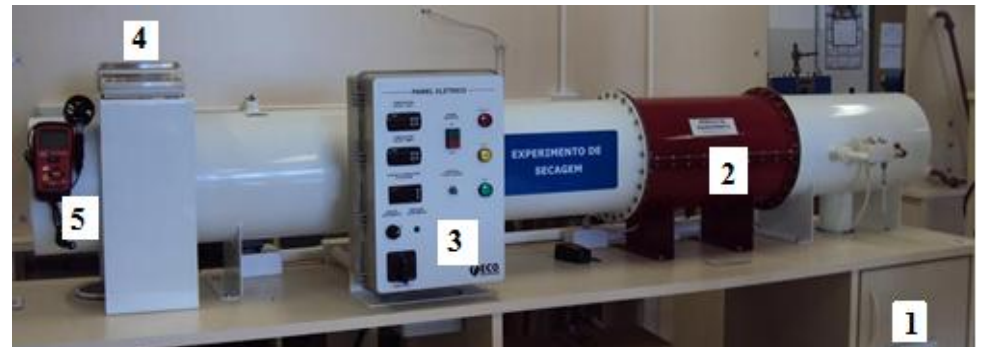

Figura 1 - Secador de leito fixo com escoamento de ar paralelo. (1) Soprador centrífugo, (2) Trocador de Calor, (3) Painel de controle, (4) Balança Digital e (5) Anemômetro de Ventoinha.

As variáveis estudadas no processo de secagem foram a temperatura ar de secagem $(T)$ de 40,50 e $60{ }^{\circ} \mathrm{C}$, a velocidade do ar ( $v$ ) em níveis de 1,0,1,5 e 2,0 m/s e altura da bandeja $(h)$ nas dimensões de 0,005, 0,075 e 0,01 m. Nas secagens a carga de sólidos na bandeja variava entre 22 e 45g, aproximadamente, conforme altura da bandeja utilizada. Para cada condição estudada, o sistema foi monitorado de 5 em 5 minutos para os primeiros 30 minutos da secagem e de 10 em 10 minutos até o sistema alcançar o equilíbrio mássico. As variáveis medidas durante os experimentos foram às temperaturas de bulbo seco $\left(T_{B S}\right)$ e bulbo úmido 
$\left(T_{B U}\right)$ do ar na entrada, a temperatura do ar de secagem $(T)$ e a massa na bandeja $(m)$. Com os valores da $T_{B S}$ e $T_{B U}$ na entrada do secador e com o valor da $T$ do ar de secagem, determinouse a umidade relativa $(U R)$ e absoluta $(U A)$ antes e após o aquecimento do ar de secagem. Para cada experimento realizado, os teores da $U_{B U}$, tanto antes como após o processo de secagem, foram obtidos através do método gravimétrico recomendado AOAC (1997). Também foram determinados para cada experimento realizado os valores de $G$ do produto final através da metodologia recomendada nas Regras para Análise de Sementes (Brasil., 1992).

\section{RESULTADOS E DISCUSSÃO}

A Tabela 1 mostra os resultados obtidos na caracterização física e fisiológica das sementes de cornichão fornecidas pela Embrapa - Pecuária Sul. Verifica-se nessa tabela que o valor de $U_{B U}$ obtido encontra-se na faixa indicada (10 a $12 \%$ ) para a comercialização de sementes (Macedo et al.; 1987). Os resultados obtidos para $d_{s}, \rho_{r}, \rho_{b}, \varepsilon, \phi$, e $\theta$ não foram comparados com a literatura devido à inexistência de dados deste tipo nas bibliografias consultadas. A $G$ apresentou um valor satisfatório e dentro dos padrões exigidos comercialmente (SEAPA - Secretaria da Agricultura do Estado).

Tabela 1 - Resultados da caracterização física e fisiológica das sementes de cornichão

\begin{tabular}{cccc}
\hline Propriedade & Valor & Propriedade & Valor \\
\hline$U_{B U}(\%)$ & $10,00 \pm 0,06$ & $\varepsilon$ & $0,37 \pm 0,01$ \\
$d_{s}(\mathrm{~mm})$ & $1,37 \pm 0,01$ & $\phi$ & $0,98 \pm 0,01$ \\
$\rho_{r}\left(\mathrm{~kg} / \mathrm{m}^{3}\right)$ & $1363,66 \pm 0,67$ & $\theta\left(^{\circ}\right)$ & $25,34 \pm 0,01$ \\
$\rho_{b}\left(\mathrm{~kg} / \mathrm{m}^{3}\right)$ & $858,28 \pm 5,30$ & $G(\%)$ & $59,00 \pm 3,33$ \\
\hline
\end{tabular}

A Tabela 2 mostra os valores dos parâmetros calculados a partir do ajuste dos modelos de isoterma de GAB, BET, Oswin, Peleg e Henderson. Também nessa tabela são demonstrados os respectivos coeficientes de determinação $\left(R^{2}\right)$ para cada modelo ajustado aos dados experimentais.

Tabela 2 - Valores dos parâmetros calculados a partir do ajuste dos modelos de GAB, BET, Oswin, Peleg e Henderson

\begin{tabular}{ccc}
\hline Modelo & Valor do parâmetro a $40{ }^{\circ} \mathrm{C}$ & $R^{2}$ \\
\hline BET & $X_{m}=0,043, C=1,244 \mathrm{E}+45, n=6,457$ & 0,964 \\
GAB & $X_{m}=0,051, C=172,256, k=0,749$ & 0,995 \\
Oswin & $a=0,085, b=0,273$ & 0,984 \\
Peleg & $k_{l}=0,088, n_{l}=0,231, k_{2}=0,117, n_{2}=4,511$ & 0,997 \\
Henderson & $a=338,364 b=2,573$ & 0,935 \\
\hline Modelo & Valor do parâmetro a $50{ }^{\circ} \mathrm{C}$ & $R^{2}$ \\
\hline BET & $X_{m}=0,038, C=1,055 \mathrm{E}+45, n=8,455$ & 0,964 \\
GAB & $X_{m}=0,045, C=133,770, k=0,815$ & 0,993 \\
Oswin & $a=0,081, b=0,330$ & 0,981 \\
Peleg & $k_{l}=0,091, n_{l}=0,306, k_{2}=0,162, n_{2}=5,933$ & 0,995 \\
Henderson & $a=107,215 b=2,058$ & 0,931 \\
\hline
\end{tabular}


Tabela 2 - Continuação

\begin{tabular}{ccc}
\hline Modelo & Valor do parâmetro a $60^{\circ} \mathrm{C}$ & $R^{2}$ \\
\hline BET & $X_{m}=0,033, C=4,172 \mathrm{E}+48, n=8,728$ & 0,938 \\
GAB & $X_{m}=0,040, C=70,328, k=0,814$ & 0,974 \\
Oswin & $a=0,070, b=0,342$ & 0,979 \\
Peleg & $k_{l}=0,098, n_{l}=0,456, k_{2}=0,235, n_{2}=11,602$ & 0,989 \\
Henderson & $a=106,787 b=1,941$ & 0,935 \\
\hline
\end{tabular}

Pela análise da Tabela 2, é possível afirmar que o modelo matemático de Peleg foi o que melhor descreveu o comportamento das isotermas de dessorção das sementes de cornichão (Lótus corniculatus L.) para as três temperaturas em estudo. Para este modelo, os coeficientes de determinação $\left(R^{2}\right)$ ficaram na ordem de 0,989 a 0,997 . Os parâmetros de ajuste para o modelo de Peleg encontrados neste estudo estão de acordo com a literatura, visto que Vásquez et al., (2013) indica valores de $n_{1}<1$ e valores de $n_{2}>1$. Na Figura 2 são apresentadas as curvas experimentais de isoterma ajustadas pelo modelo de Peleg.

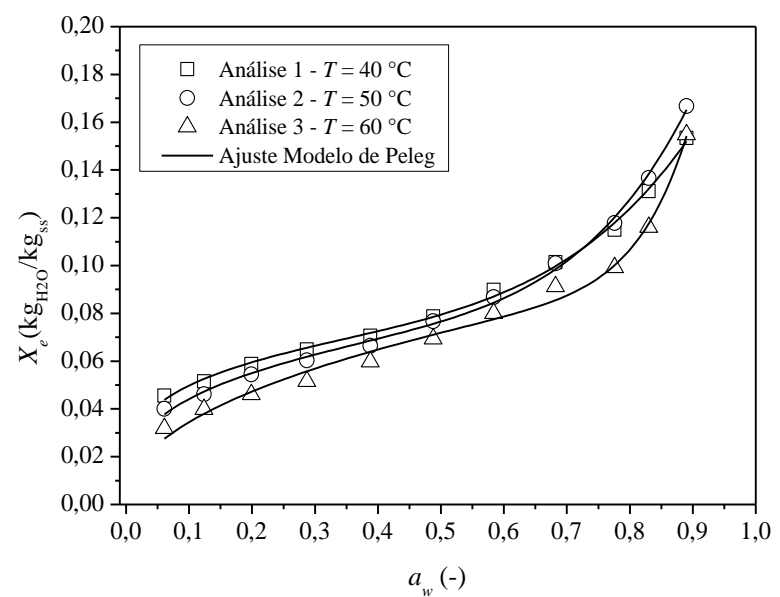

Figura 2 - Umidade de equilíbrio $\left(X_{e}\right)$ em função da atividade de água $\left(a_{w}\right)$.

Através da análise do comportamento da isoterma apresentada na Figura 2, é verificada que esta é do tipo II, seguindo a classificação de Brunauer de 1938. A forma sigmoidal da classificação do tipo II é característica de isotermas de produtos orgânicos (Vega et al., 2005). Este tipo de isoterma resulta de adsorventes nos quais há uma extensa variação de tamanhos de poros. Esta variação permite ao adsorvente a capacidade de avançar de forma contínua de uma adsorção monocamada para multicamada, seguida de condensação capilar. Observa-se também que o aumento da $a_{w}$ promove um aumento da $X_{e}$ para cada temperatura. Sendo assim, a taxa de dessorção é mais alta no início do processo e diminui continuamente à medida que se aproxima da $X_{e}$ (Palmeira et al., 2004).

Na Figura 3 são apresentadas as curvas do adimensional de água livre em função do tempo para todas as secagens realizadas neste trabalho. Do mesmo modo, são apresentados na Tabela 3 os valores obtidos para a umidade final e germinação total das sementes secas. 


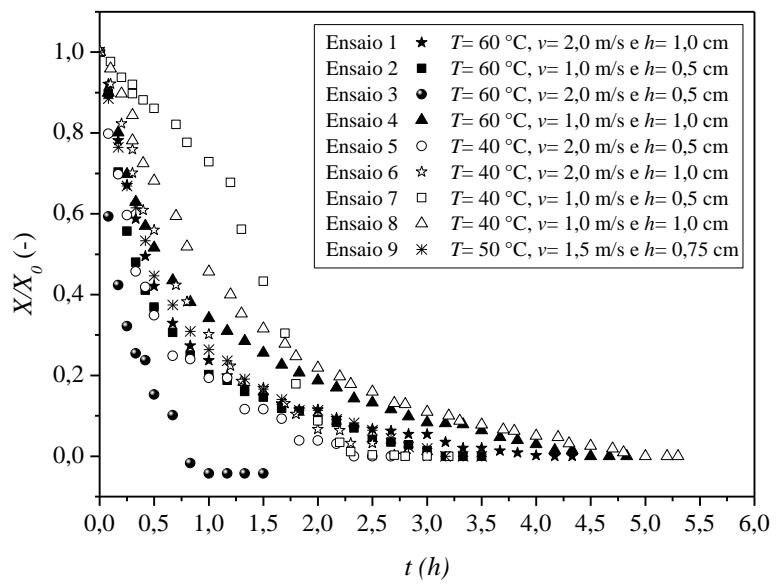

Figura 3 - Adimensional de água livre em função do tempo.
Tabela 3 - Umidade final e germinação total das sementes secas

\begin{tabular}{ccc}
\hline Ensaio & $U_{B U}(\%)$ & $G(\%)$ \\
\hline 1 & $5,06 \pm 0,01$ & $57,0 \pm 2,0$ \\
2 & $5,68 \pm 0,04$ & $55,0 \pm 2,0$ \\
3 & $7,34 \pm 0,15$ & $52,3 \pm 5,5$ \\
4 & $5,35 \pm 0,04$ & $49,3 \pm 1,5$ \\
5 & $9,04 \pm 0,11$ & $53,3 \pm 2,4$ \\
6 & $8,95 \pm 0,12$ & $44,0 \pm 7,3$ \\
7 & $7,01 \pm 1,28$ & $48,6 \pm 5,1$ \\
8 & $8,44 \pm 0,01$ & $59,0 \pm 4,0$ \\
9 & $6,79 \pm 0,07$ & $47,4 \pm 3,1$ \\
\hline
\end{tabular}

Verifica-se na Figura 3 que todas as curvas apresentaram um comportamento característico de secagem, com diminuição do teor de água livre em função do tempo. Nessa figura, ao se analisar a influência da temperatura e da velocidade do ar de secagem, é observado que o aumento dessas variáveis promoveu uma diminuição do tempo de secagem. Já o aumento da altura da bandeja promoveu um aumento do tempo de secagem. Este resultado condiz com o esperado fisicamente. $\mathrm{O}$ aumento da temperatura e da velocidade do ar promove um aumento da força motriz do processo de secagem, facilitando assim a transferência de calor e de massa do sistema. Por outro lado, o aumento da altura de bandeja provoca uma resistência maior ao processo de secagem, o que dificulta a transferência de calor e de massa do sistema.

Analisando os resultados encontrados na Tabela 3, verificou-se que os resultados obtidos para a $U_{B U}$ das sementes secas ficaram na faixa de $5 \%$ para as secagens realizadas a $60{ }^{\circ} \mathrm{C}$ e em aproximadamente $8 \%$ para as secagens realizadas a $40{ }^{\circ} \mathrm{C}$. Este resultado está de acordo com o esperado fisicamente. Uma vez que o aumento da temperatura promove uma diminuição na umidade relativa do ambiente, é esperado que a retirada da umidade seja mais efetiva nas temperaturas maiores. No que se refere aos $G$, é observado na Tabela 3 que os valores encontrados estão na ordem de 44 a $59 \%$ para as secagens realizadas neste estudo. Dentre as secagens, a que obteve o maior valor de $\mathrm{G}$, com valor de $59 \%$, foi a realizada na condição de $T=40{ }^{\circ} \mathrm{C}, v=1,0 \mathrm{~m} / \mathrm{s}$ e $h=1,0 \mathrm{~cm}$. Esse valor é muito próximo ao obtido nas sementes fornecidas pela Empresa Brasileira de Pesquisa Agropecuária - Embrapa Pecuária Sul da cidade de Bagé/RS e indica que esta secagem não modificou a qualidade fisiológica das sementes.

\section{CONCLUSÕES}

$\mathrm{Na}$ caracterização física das sementes foi possível obter-se um valor do diâmetro de Sauter de 1,37 $\pm 0,01 \mathrm{~mm}$, de massa específica real de $1363,66 \pm 0,67 \mathrm{~kg} / \mathrm{m}^{3}$, de massa específica bulk de $858,28 \pm 5,30 \mathrm{~kg} / \mathrm{m}^{3}$, de porosidade de $0,37 \pm 0,01$, de esfericidade de 0,98 $\pm 0,01$, ângulo de repouso de $25,34 \pm 0,01^{\circ}$, de umidade em base úmida de $10,00 \pm 0,06 \%$ e germinação de $59,00 \pm 3,33 \%$. 
No estudo das isotermas de dessorção, pode-se concluir que a forma da isoterma na qual as sementes de cornichão (Lotus corniculatus L.) seguem é a do tipo II da classificação de Brunauer de 1938 e que o modelo matemático que melhor descreve seu comportamento foi o modelo de Peleg com um $R^{2}$ na ordem de 0,989 a 0,997 .

Para o estudo da secagem das sementes reumidificadas pode-se concluir que todas as condições experimentais estudadas neste trabalho possibilitaram colocar os índices de umidade final das sementes nas condições exigidas comercialmente (abaixo de $10 \%$ ). $\mathrm{Na}$ análise do adimensional de água livre em função do tempo concluiu-se que um aumento na altura da bandeja proporcionou uma diminuição no processo de secagem. Pode-se também concluir através destes adimensionais, que a temperatura e a velocidade do ar influenciam no processo de secagem aumentando a força motriz do processo.

Finalmente, conclui-se que para as condições de secagem estudadas neste trabalho os testes fisiológicos das sementes secas apresentaram índices de germinação na ordem de 44 a $59 \%$, sendo o maior valor obtido na secagem realizada na condição de $T=40^{\circ} \mathrm{C}, v=1,0 \mathrm{~m} / \mathrm{s}$ e $h=1,0 \mathrm{~cm}$. Esse valor, que é muito próximo ao obtido nas sementes fornecidas pela Empresa Brasileira de Pesquisa Agropecuária - Embrapa Pecuária Sul da cidade de Bagé/RS, indica que esta secagem não modificou a qualidade fisiológica das sementes.

\section{REFERÊNCIAS}

AOAC, Association of Oficial Analytical Chemists. Official methods of analysis. 16th ed.Washington D.C.: AOAC, v.2, 1997.

ARNOSTI JR., S. Desenvolvimento de um Secador Transportador do Tipo Esteira com Escoamentos Cruzados e Caracterização de Sementes de Gramíneas. Programa de PósGraduação em Engenharia Química (Doutorado), p.232, 1997.

BLAHOVEC, J. Sorption isotherms in materials of biological origin mathematical and physical approach. Journal of Food Engineering, v.65, p.489-495, 2004.

BRASIL, M. A. R. A. Regras para Análise de Sementes. Brasília: SNDA/DNDV/CLAV, p.365, 1992.

CHEN, C.; JAYAS, D. S. Evaluation of the GAB equation for the isotherms of agricultural products. Transactions of ASAE, v.41, n 6, p.1755-1760, 1998.

CURTIS, K.; MCKERSIE, B. O. Growth potential of the axis as a determinant of seeding vigor in birdsfoot trefoil crop science, v.24, p.47-50, 1984.

ETTLIN, W. H.; LAVERACK, G. Seed quality in Lotus corniculatus in relation to pod maturity and harvest treatment. Lotus Newsletter, v. 27, 1996.

FERRARI FILHO, E. Métodos e temperaturas de secagem sobre a qualidade físico-química e microbiológica de grãos de milho no armazenamento. Universidade federal do Rio Grande do Sul, Programa de Pós-graduação em Fitotecnia (Mestrado), p.5, 2011.

FIGUEIRÊDO, R. M. F.; QUEIROZ, A. J. M. Isotermas se adsorção de umidade da pitanga em pó. Revista de Biologia e Ciências da Terra, v.7, $\mathrm{n}^{\circ}$ 1, 2007. 
GONELI, A. L. D. Variação das propriedades físico-mecânicas e da qualidade da mamona (Ricinus communis L.) durante a secagem e o armazenamento. Universidade Federal de Viçosa (Doutorado), p.199, 2008.

MACEDO, G. A. R.; MARQUES NETO, J. E.; BATISTA, J. S. Secagem à sombra e ao sol de sementes de gramíneas forrageiras. Revista Brasileira de Sementes. Ano 9, $\mathrm{n}^{\mathrm{o}} 3$, p. 29-37, 1987.

MELO, P. T. B. S.; BARROS, A. C. A. S. Estudo sobre o consumo de trevo branco (Trifolium repens L.), cornichão (Lotus corniculatus L.) e cornichão El Rincon (Lotus subbiflorus Lag.) no Rio Grande do Sul. Revista Brasileira Agrociência, v.9, $\mathrm{n}^{\circ}$ 3, p. 291-295, 2003.

MOREY, V. W. F.; MERONUCK, R. A.; LANG, J. P. Relationship between equilibrium relative humidity and deterioration of shelled corn. Transactions of ASAE, v. 38, $\mathrm{n}^{\circ} 4$, p. 1139-1145, 1995.

MOTA, F. S.; BERNY, Z. B.; MOTA, J. F. A. S. Índice climático de crescimento de pastagens naturais no Rio Grande do Sul. Pesquisa Agropecuária Brasileira, Brasília, v. $16, \mathrm{n}^{\circ} 4, \mathrm{p} .453-472,1981$.

PALMEIRA, J.; SILVA, E.; CARDOSO, F.; DO NASCIMENTO, J.; DA SILVA, M. Isotermas de dessorcao em frutos de cajá (Spondia lútea L.). Revista Brasileira de almecenamiento, v.29, $\mathrm{n}^{\circ}$ 2, p.76-81, 2004.

SAMANIEGO-ESGUERRA, C. M.; BOAG, I. F.; ROBERTSON, G. L. Comparison of regression methods for fitting the Gab model to the moisture isotherms of some dried fruit and vegetables. In: VIEIRA, H. A.; FIGUEIRÊDO, R. M. F.; QUEIROZ, A. J. M. Isotermas se adsorção de umidade da pitanga em pó. Revista de Biologia e Ciências da Terra, Campina Grande, v.7, n 1, 2007.

STRUMILLO, C. Z.; KUDRA, T. Drying: principles, applications and design. In: HUGHES, R. Topics in chemical engineering. UK: Gordon and Breach Science Publisher, v.3, 1986. In: OLIVEIRA, E.G. Secagem de Spirulina platensis: análise das técnicas de leito de jorro e camada delgada. Dissertação (Mestrado), Faculdade de Engenharia Química, Fundação Universidade Federal do Rio Grande, 2006.

VIANDS, D. R.; EHLKE, N. J.; PAPADOUlOS, Y. A. Cooperative Project to develop birdsfoot trefoil with multiple desease resistence. Newsletter, v. 25, 1994.

VÁSQUEZ, C.; DIAZ-CALDERON, P.; ENRIONE, J.; MATIACEVICH, S. State diagram, sorption isotherm and color of blueberries as a function of water content. Journal of Food Engineering, n ${ }^{\circ}$ 520, p.8-15, 2013.

VEGA, A.; ANDRES, A.; FITO, P. Modelado de la cinética de secado del pimiento Rojo (capsicum annuum L.). Información tecnológica, $\mathrm{n}^{\circ}$ 16, p.3-11, 2005.

\section{AGRADECIMENTOS}

Os autores deste trabalho agradecem a UNIPAMPA/Bagé e EMBRAPA/ Pecuária Sul pela infraestrutura disponibilizada e ao CNPq e a CAPES pela concessão das bolsas. 